\title{
On the accuracy of the Derjaguin approximation for depletion potentials
}

\author{
S.M. Oversteegen*, H.N.W. Lekkerkerker \\ Van 't Hoff Laboratory for Physical and Colloid Chemistry, Debye Research Institute, \\ Utrecht University, P.O. Box 80051, 3508 TB Utrecht, The Netherlands
}

Received 28 February 2004

\begin{abstract}
The accuracy of the Derjaguin expressions for the depletion potential of large spheres due to small spheres, discs, and rods is analysed. To that end, we subdivide the generalised Gibbs adsorption equation into three contributions. We determine these contributions both in first order of curvature of the large spheres as well as exact for small spheres, discs, and rods up to first order in the number density of the depletion agents. The gain of volume in the bulk when the depletion zones of the large spheres overlap is the same for spheres, discs, and rods of equally characteristic sizes. The Derjaguin approximation underestimates the exact solution because of the neglected curvature. The number of particles that fit in the gap between the two large spheres as well as the amount that can enter a single depletion zone, are both underestimated by the Derjaguin approximation for discs and rods to relatively the same extent. Due to the intermediate excluded volume of the discs, the correction of the last two contributions just cancels the error in the first one. Small spheres only exhibit the first contribution, whereas for rods the other parts overcompensate the first. We can thus explain why the depletion potential of large spheres due to small spheres is underestimated by the Derjaguin approximation, is surprisingly accurate for discs, and is overestimated for rod-like depletion agents.
\end{abstract}

(C) 2004 Elsevier B.V. All rights reserved.

PACS: 05.70.Ce; 64.70.Nd; 82.70.Dd

Keywords: Depletion interaction; Colloids

\footnotetext{
* Corresponding author.

E-mail address: m.oversteegen@chem.uu.nl (S.M. Oversteegen).
} 


\section{Introduction}

It is generally believed that in mixed colloidal suspensions containing colloids that differ in size or shape, the mutual asymmetry may bring about a nett attraction by the so-called depletion effect. This depletion effect has been established for bimodal mixtures hard spheres both experimentally [1-6] and theoretically [7-14]. When two large spheres of diameter $\sigma$ approach each other up to a distance $h$ smaller than the diameter of a smaller sphere, $a$, the latter is expelled from the gap. Consequently, there is an unbalanced osmotic pressure inside and outside the gap which gives rise to a nett attraction. The resulting depletion potential for $a \ll \sigma$ up to first order in the number density of the smaller spheres, $n_{s}$, follows from the Derjaguin approximation as $[15,16]$

$$
\frac{W_{\text {spheres }}}{k_{B} T}=-\frac{\pi}{4} n_{s} a^{2} \sigma\left(1-\frac{h}{a}\right)^{2} \text {. }
$$

Up to first order in the number density of small spheres the exact depletion potential can also be calculated analytically [17]. From which it is established that the Derjaguin approximation underestimates the depth of the potential.

An entropically driven phase-separation is also expected to be found for colloidal mixtures of hard spheres with platelets. The orientation of platelets is restricted when confined between two spheres. Approximating the platelets by discs, i.e., infinitely thin of diameter $D$, the depletion potential for such systems up to first order in the number density of discs, $n_{p}$, reads by applying the Derjaguin approximation [18]

$$
\begin{aligned}
\frac{W_{\text {discs }}}{k_{B} T}= & -\frac{\pi}{6} n_{p} D^{2} \sigma\left[\frac{3}{2} \frac{h}{D} \arcsin \frac{h}{D}-\frac{3}{4} \pi \frac{h}{D}\right. \\
& \left.+\left(1+\frac{1}{2}\left(\frac{h}{D}\right)^{2}\right) \sqrt{1-\left(\frac{h}{D}\right)^{2}}\right] .
\end{aligned}
$$

We have previously shown from calculations [19] that this potential is remarkably accurate, slightly overestimating the true potential. Going to platelets of finite thickness, e.g., oblate ellipsoids, the deviation from the Derjaguin approximation may change sign [18].

Analogous to platelets, the orientational entropy of infinitely thin hard rods of length $L$ decreases when placed between two spheres. This has been established both by experiments [20-22] and theory [23-25]. The decreased number of conformations results a pressure deficit which leads to a depletion potential that, by using the Derjaguin approximation, up to first order in the number density, $n_{r}$, is given by [26-28]

$$
\frac{W_{\text {rods }}}{k_{B} T}=-\frac{\pi}{12} n_{r} L^{2} \sigma\left(1-\frac{h}{L}\right)^{3} .
$$

The error introduced by the Derjaguin approximation for the depletion of large spheres due to rods has been calculated [29]. It now turns out that the Derjaguin approximation overestimates the potential. Within the appropriate limits the Derjaguin potential, Eq. (3) turns out to be quite accurate from experiments [30-32]. 
Although the Derjaguin approximation has been applied in the same way to small spheres, rods, and platelets between two large spheres, the accuracy of the approximation differs with the shape of the depletion agent. In this paper we will show where this difference stems from.

The 'classic' Derjaguin approach [33] is to determine the interaction per unit area between two parallel planar walls first. Subsequently, this interaction $w(h)$ is integrated in order to account for the curvature of the two large spheres. For spheres of diameter $\sigma$ this procedure yields

$$
W_{\text {Derj }}(h)=\frac{1}{2} \pi \sigma \int_{h}^{\infty} w\left(h^{\prime}\right) \mathrm{d} h^{\prime} .
$$

In spite of the fact that this method provides us with the depletion potentials as quoted earlier, it does not give a clue on what determines the accuracy of the potential for different shapes. To make progress, we subdivide the depletion potential that arises from the generalised Gibbs adsorption equation into three contributions. Subsequently, we derive the above Derjaguin equations as well as exact expressions for these contributions. Upon comparison of the three contributions we finally show how the error in the depletion potentials from Derjaguin's approximation is introduced.

\section{Contributions to the depletion potential}

Consider two hard objects at a nearest face-to-face-separation $h$ that are in equilibrium with a reservoir of depletion agents with chemical potential $\mu$. For fixed volume and temperature, the mentioned variables completely describe the system. Hence, the appropriate thermodynamic potential is given by

$$
\mathrm{d} \Psi=-f \mathrm{~d} h-N \mathrm{~d} \mu .
$$

Here $N$ is the (ensemble averaged) number of depletion agents in the system. The depletion force $f$ is related to the depletion potential $W$ by

$$
f=-\left(\frac{\partial W}{\partial h}\right)_{\mu} \text {. }
$$

From the total differential equation (5) we obtain, using Eq. (6), the Maxwell relation

$$
\left(\frac{\partial N}{\partial h}\right)_{\mu}=\left(\frac{\partial f}{\partial \mu}\right)_{h}=-\left(\frac{\partial}{\partial h}\left(\frac{\partial W}{\partial \mu}\right)_{h}\right)_{\mu} .
$$

Since the depletion potential $W$ vanishes at infinite separation for all values of the chemical potential, it is found after integration with respect to $h$ that [34-37]

$$
-\left(\frac{\partial W}{\partial \mu}\right)_{h}=N(h)-N(\infty) \text {. }
$$

This is the generalised Gibbs-equation. 


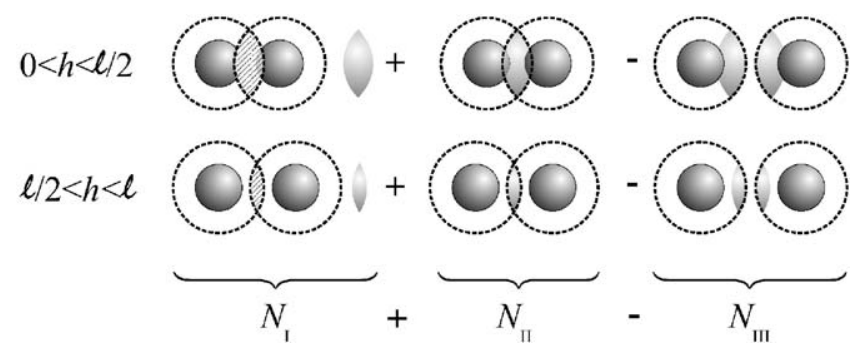

Fig. 1. Schematic representation of the three contributions to the depletion potential between two spheres. Two situations must be distinguished: one where the overlap volumes also coincides with the spheres themselves (top) and one that the depletion zones only overlap each other (bottom).

In the following we will only consider dilute suspensions, so we can write for the chemical potential

$$
\mu=\mu^{\circ}+k_{B} T \ln n_{b},
$$

where $n_{b}$ is the bulk number density of the depletion agents that coexists with the reservoir. Moreover, the number of particles $N$ is up to first order linear in $n_{b}$. Consequently, integration of Eq. (8) with respect to the chemical potential finally yields for the depletion potential of two objects due to depletion agents

$$
\frac{W(h)}{k_{B} T}=-(N(h)-N(\infty))
$$

Eq. (9) is exact up to first order in the density and allows us to connect it to the depletion effect in the following way. The characteristic size $\ell / 2$ is the largest distance a depletion agent can just touch one of the objects. Here, $\ell$ is the diameter $a$ of a small sphere, the length $L$ of a rod, or the diameter $D$ of a disc. At distances less than $\ell / 2$ the depletion agent is hindered in its conformational freedom. That region is therefore called the taboo or depletion zone. When depletion zones of the two objects start to overlap, i.e., $h<\ell$, extra accessible volume is gained in bulk. Moreover, particles in the depletion zone that already were hindered by one of the objects, may be hindered extra by the other object. Hence, the contribution to the depletion potential is threefold.

The first contribution, $N_{I}$, represents the number of particles that access the volume in the bulk that has become available by the overlap of depletion zones. A second part of the depletion potential, $N_{I I}$, is the number of particles that still fit into the overlapping zones when the two objects are a distance $h$ apart. Finally, $N_{I I I}$ accounts for the number of particles that would have been in the overlap volume when the two objects are infinitely apart, i.e., $h>\ell$, so the depletion agent is only hindered by one object. The three contributions are displayed schematically in Fig. 1 for large spheres.

Clearly, $N_{I I}$ contributes to $N(h)$ in Eq. (9), whereas $N_{I I I}$ has its share in $N(\infty)$. However, $N_{I}$ participates in both terms. We therefore may rewrite Eq. (9) as

$$
\frac{W}{k_{B} T}=-\left(N_{I}+N_{I I}-N_{I I I}\right) \text {. }
$$

The explicit face-to-face-distance dependence $h$ has been dropped for convenience. 
In the remainder of this paper we will consider the two depleted objects to be large hard spheres. We determine for small spherical, plate-like, and rod-like depletion agents the three contributions exactly as well as by means of the Derjaguin approximation. We can thus resolve what term determines the error of the latter.

\section{Depletion potential for bimodal mixtures of spheres}

Let us first consider small hard spheres of diameter $\ell=a$ to be the depletion agents of the two large hard spheres with a size $\sigma$ each.

\subsection{Derjaguin approximation}

The first contribution to the depletion potential is the number of particles that can freely move in bulk in the volume that has become available as soon as the depletion zones start to overlap. Hence,

$$
N_{I}=n_{b} V_{\text {overlap }},
$$

where $n_{b}$ is the bulk number density and $V_{\text {overlap }}$ is the volume of the overlapping depletion zones. The volume of a cap of height $H$ of a sphere of radius $R$ is given by $\frac{\pi}{3} H^{2}(3 R-H)$. Using $H=\frac{1}{2}(\ell-h)$ and $R=\frac{1}{2}(\ell+\sigma)$, as can be seen on the left of Fig. 1, we obtain for $0 \leqslant h \leqslant \ell$

$$
N_{I}=n_{b} \frac{2}{3} \pi\left(\frac{\ell}{2}-\frac{h}{2}\right)^{2}\left(\frac{3 \sigma}{2}+\ell+\frac{h}{2}\right) .
$$

Rearranging terms in Eq. (12) yields

$$
N_{I}=n_{b} \frac{\pi}{4} \sigma \ell^{2}\left\{\left(1-\frac{h}{\ell}\right)^{2}+\frac{2}{3} \frac{\ell}{\sigma}-\frac{h}{\sigma}\left(1-\frac{1}{3}\left[\frac{h}{\ell}\right]^{2}\right)\right\} \text {. }
$$

In most textbooks (see, e.g., [38-40]) the Derjaguin expression, Eq. (4), is derived by expanding the curvature of the large sphere to second order. Hence, the Derjaguin approach becomes exact for infinitely large spheres and is thought to be a good approximation when the spheres are significantly larger than the depletion agents, i.e., $\sigma \gg \ell$. In the remainder we take the last-mentioned property for the Derjaguin approximation. Since $0 \leqslant h \leqslant \ell$, we may also take $h \ll \sigma$. That is, the second and third term in braces of Eq. (13) vanish. We now find

$$
N_{I}=n_{b} \frac{\pi}{4} \sigma \ell^{2}\left(1-\frac{h}{\ell}\right)^{2} \text {. }
$$

Since we are considering hard spheres, the small spheres are not able to enter the gap between two large spheres that are $0 \leqslant h \leqslant a$ apart. Moreover, the small spheres cannot come closer to a single sphere than its radius $a / 2$. Consequently, the two other contributions to the depletion potential are found trivially as

$$
N_{I I}=N_{I I I}=0 \text {. }
$$


Insertion of Eqs. (14) and (15) into Eq. (10) using $\ell=a$ finally yields for the depletion potential of a bimodal mixture of spheres

$$
\frac{W_{\text {spheres }}}{k_{B} T}=-n_{s} \frac{\pi}{4} a^{2} \sigma\left(1-\frac{h}{a}\right)^{2} .
$$

This is indeed the Derjaguin expression for the depletion potential as already given by Eq. (1).

\subsection{Exact solution}

The exact solution for the depletion potential for bimodal mixtures of hard spheres up to first order in density can be found straightforwardly from the previous results. The first contribution to the depletion potential, $N_{I}$, is given by Eq. (13). The other two contributions, $N_{I I}$ and $N_{I I I}$, are found from the same reasoning that lead to Eq. (15), that is, they are both equal to zero. We therefore find

$$
\frac{W_{\text {spheres }}}{k_{B} T}=-n_{s} \frac{\pi}{4} \sigma a^{2}\left\{\left(1-\frac{h}{a}\right)^{2}+\frac{2}{3} \frac{a}{\sigma}-\frac{h}{\sigma}\left(1-\frac{1}{3}\left[\frac{h}{a}\right]^{2}\right)\right\} .
$$

It is readily seen that this is the same expression for the depletion potential as previously has been found for the depletion of spheres due to penetrable hard spheres by means of a force method [17]. Indeed, up to first order in the density hard spherical depletion agents give the same results as hard penetrable hard spheres [41-43].

\section{Depletion potential for mixtures of spheres and discs}

In this section we will study the depletion potential of two hard spheres of diameter $\sigma$ due to infinitely thin hard platelets, i.e., discs of diameter $\ell=D$.

\subsection{Derjaguin approximation}

The number of discs that can freely move in the available volume in the bulk follows again from Eq. (11). In our Derjaguin approximation, this contribution is therefore given by Eq. (14) with $\ell=D$

$$
N_{I}=n_{p} \frac{\pi}{6} \sigma D^{2} \times \frac{3}{2}\left(1-\frac{h}{D}\right)^{2} .
$$

In contrast to small hard spheres, the discs are able to enter the gap between the two large spheres for distances $0 \leqslant h \leqslant D$. The number of discs that still fit into the gap can formally be written as

$$
N_{I I}=2 \int_{\max \left(0, h-\frac{1}{2} D\right)}^{D / 2} A(x) n(x) \mathrm{d} x .
$$

Here $A(x)$ is the area of a spherical cap of the overlap volume at a distance $x$ from the surface of the depleted large sphere. The local number density at that distance is given 


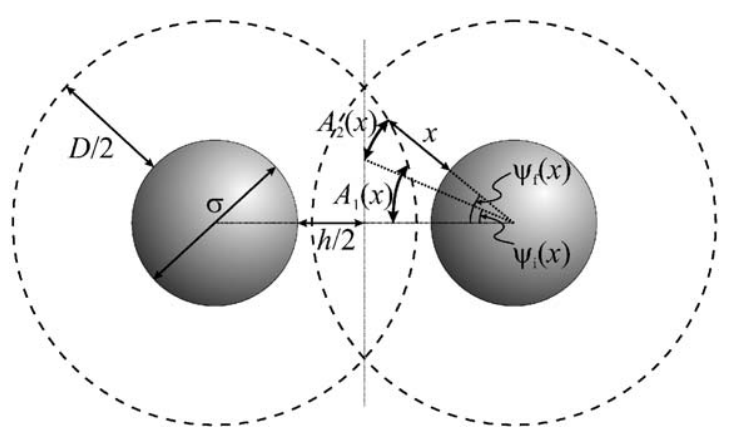

Fig. 2. The area between the horizontal symmetry axis and the surface of the overlap volume at a distance $x$ is given by $A_{1}(x)\left(\max \left(0, h-\frac{1}{2} D\right) \leqslant x \leqslant \frac{1}{2} h\right)$, whereas $A_{2}(x)$ is bounded by the vertical symmetry axis and the outer shell of that volume $\left(\frac{1}{2} h<x \leqslant D / 2\right)$. The initial and final opening angle of those areas are denoted as $\psi_{i}(x)$ and $\psi_{f}(x)$, respectively.

by $n(x)$. The factor 2 comes in since it applies for both spheres symmetrically. The lower limit of the integral depends on whether the overlap volume overlaps the adjacent sphere (upper line in Fig. $1,0 \leqslant h \leqslant D / 2$ ) or not (lower line in Fig. $1, D / 2<h \leqslant D$ ). In the former case the overlap volume starts at the surface of the sphere $(x=0)$, whereas in the latter it begins at $x=h-\frac{1}{2} D$.

The area $A(x)$ can mathematically be determined from

$$
\begin{aligned}
A(x) & =2 \pi\left(\frac{1}{2} \sigma+x\right)^{2} \int_{\psi_{i}(x)}^{\psi_{f}(x)} \sin \psi \mathrm{d} \psi \\
& =2 \pi\left(\frac{1}{2} \sigma+x\right)^{2}\left(\cos \psi_{i}(x)-\cos \psi_{f}(x)\right),
\end{aligned}
$$

where $\psi_{i}(x)$ is the initial angle relative to the horizontal symmetry axis and $\psi_{f}(x)$ is the final opening angle, as it can be seen from Fig. 2. As it can also be seen from Fig. 2, we have to distinguish between two regions for the area $A(x)$. That is, one area is bounded on one side by the horizontal symmetry axis $\left(\max \left(0, h-\frac{1}{2} D\right) \leqslant x \leqslant \frac{1}{2} h\right)$ and another area by the vertical axis $\left(\frac{1}{2} h<x \leqslant D / 2\right)$. Both areas are bounded on the other side by the outer surface of the overlap volume.

Using Fig. 2, elementary trigonometry provides us with

$$
\begin{aligned}
& \cos \psi_{i}(x)= \begin{cases}1, & \max \left(0, h-\frac{1}{2} D\right) \leqslant x \leqslant \frac{1}{2} h \\
\frac{\sigma+h}{\sigma+x}, & \frac{1}{2} h<x \leqslant \frac{1}{2} D\end{cases} \\
& \cos \psi_{f}(x)=\frac{(\sigma+h)^{2}+\left(\frac{1}{2} \sigma+x\right)^{2}-\frac{1}{4}(\sigma+D)^{2}}{(\sigma+h)(\sigma+2 x)} .
\end{aligned}
$$




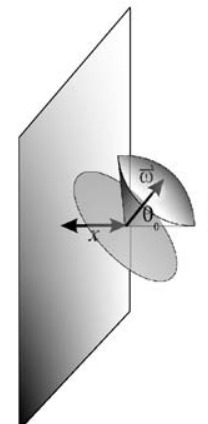

(a)

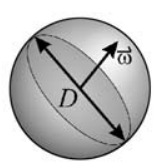

Fig. 3. (a) In the bulk the director $\vec{\omega}$ of a disc with diameter $D$ can describe a full unit sphere (right), whereas a disc at a distance $x<D / 2$ to a wall only describes part of it (left). (b) The azimuth $\theta_{0}$ between the director $\vec{\omega}$ and the normal on a planar wall $\vec{n}$ follows from $\sin \theta_{0}=x /(D / 2)$.

Insertion of Eqs. (21) and (22) into Eq. (20) gives after some algebra

$$
\begin{aligned}
A_{1}(x)= & \pi \sigma \frac{\frac{1}{2} \sigma+x}{\sigma+h}\left(\left(x-h+\frac{1}{2} D\right)-\frac{(h-x)^{2}}{\sigma}+\frac{D^{2}}{4 \sigma}\right), \\
& \max \left(0, h-\frac{1}{2} D\right) \leqslant x \leqslant \frac{1}{2} h, \\
A_{2}(x)= & \frac{1}{2} \pi \sigma \frac{\frac{1}{2} \sigma+x}{\sigma+h}\left((D-2 x)+\frac{D^{2}}{4 \sigma}-\frac{x^{2}}{\sigma}\right), \quad \frac{1}{2} h<x \leqslant \frac{1}{2} D .
\end{aligned}
$$

In our approach of the Derjaguin approximation, $D \ll \sigma$ and therefore $\{x, h\} \ll \sigma$, we find

$$
\begin{aligned}
& A_{1}(x) \approx \frac{1}{2} \pi \sigma\left(x-h+\frac{1}{2} D\right), \quad \max \left(0, h-\frac{1}{2} D\right) \leqslant x \leqslant \frac{1}{2} h, \\
& A_{2}(x) \approx \frac{1}{4} \pi \sigma(D-2 x), \quad \frac{1}{2} h<x \leqslant \frac{1}{2} D .
\end{aligned}
$$

We also require the number density profile $n(x)$ in order to solve Eq. (19). We will henceforth assume that the density profile between two planar walls also applies for spherical interfaces within the aforementioned limit, i.e., $D \ll \sigma$. The director $\vec{\omega}$ of a disc can rotate freely in bulk and can assume all polar angles. However, in the vicinity of the wall the angle of the director is limited to $0 \leqslant \theta \leqslant \theta_{0}$, where $\theta$ is the angle between the normal of the disc and that of the wall. As it follows from Fig. 3, the azimuth follows from $\sin \theta_{0}=\frac{x}{D / 2}$. Hence, the number density compared to that in the bulk is to first order given by

$$
\frac{n(x)}{n_{p}}=\int_{0}^{\theta_{0}} \sin \theta \mathrm{d} \theta=1-\cos \theta_{0}=1-\sqrt{1-\left(\frac{x}{D / 2}\right)^{2}} .
$$

If we put Eq. (27) for the number density and Eqs. (25) and (26) for the area into Eq. (19), we finally obtain after integration for the two situations of the lower 
integration limit

$$
N_{I I}=\left\{\begin{array}{l}
n_{p} \frac{\pi}{6} \sigma D^{2}\left\{\frac{1}{4}-\frac{3}{8} \pi-\frac{3}{2}\left(\frac{h}{D}\right)^{2}+\left(1+\frac{1}{2}\left(\frac{h}{D}\right)^{2}\right)\right. \\
\left.\quad \times \sqrt{1-\left(\frac{h}{D}\right)^{2}}+\frac{3}{2}\left(\frac{h}{D}\right) \arcsin \left(\frac{h}{D}\right)\right\}, \quad 0 \leqslant h \leqslant \frac{1}{2} D \\
n_{p} \frac{\pi}{6} \sigma D^{2}\left\{\frac{3}{2}\left(1-\frac{h}{D}\right)^{2}-\frac{3}{8} \pi+\left(2 \frac{h}{D}\left(1-\frac{h}{D}\right)-\frac{3}{2}\right)\right. \\
\quad \times \sqrt{\frac{h}{D}\left(1-\frac{h}{D}\right)}+\left(1+\frac{1}{2}\left(\frac{h}{D}\right)^{2}\right) \sqrt{1-\left(\frac{h}{D}\right)^{2}}+\frac{3}{2} \frac{h}{D} \\
\left.\quad \times \arcsin \left(\frac{h}{D}\right)-\frac{3}{4}\left(2 \frac{h}{D}-1\right) \arcsin \left(2 \frac{h}{D}-1\right)\right\}, \quad \frac{1}{2} D<h \leqslant D .
\end{array}\right.
$$

The number of discs that can enter the depletion zone of two single free spheres is formally given by the same equation as between two spheres, Eq. (19). However, the vertical symmetry line is no longer present, so the area is only given by $A_{1}(x)$ from Fig. 2. Hence,

$$
N_{I I I}=2 \int_{\max \left(0, h-\frac{1}{2} D\right)}^{D / 2} A_{1}(x) n(x) \mathrm{d} x .
$$

Although the exact number density profile differs from that between two spheres, within the Derjaguin approximation we take it again to be that of a planar wall. So, by taking Eq. (23) for the area and Eq. (27) for the number density profile, we find for the two different overlap situations

$$
N_{I I I}=\left\{\begin{array}{l}
n_{p} \frac{\pi}{6} \sigma D^{2}\left\{\frac{7}{4}-\frac{3}{8} \pi+\left(\frac{3}{4} \pi-3\right) \frac{h}{D}\right\}, \quad 0 \leqslant h \leqslant \frac{1}{2} D \\
n_{p} \frac{\pi}{6} \sigma D^{2}\left\{3\left(1-\frac{h}{D}\right)^{2}+\frac{3}{8} \pi\left(2 \frac{h}{D}-1\right)\right. \\
\quad+\left(2 \frac{h}{D}\left(1-\frac{h}{D}\right)-\frac{3}{2}\right) \sqrt{\frac{h}{D}\left(1-\frac{h}{D}\right)} \\
\left.\quad-\frac{3}{4}\left(2 \frac{h}{D}-1\right) \arcsin \left(2 \frac{h}{D}-1\right)\right\}, \quad \frac{1}{2} D<h \leqslant D .
\end{array}\right.
$$

Before inserting Eqs. (28) and (30) into Eq. (10), we notice that $N_{I}$ is, unlike $N_{I I}$ and $N_{I I I}$, independent on the two different overlap situations. Moreover, the depletion potential Eq. (2) is neither subjected to such parting. The difference $N_{I I}-N_{I I I}$ therefore should not hinge on this distinction. We indeed find from both Eqs. (28) and (30)

$$
\begin{aligned}
N_{I I}-N_{I I I}= & \frac{\pi}{6} n_{p} D^{2} \sigma\left\{-\frac{3}{2}\left(1-\frac{h}{D}\right)^{2}-\frac{3}{4} \pi \frac{h}{D}+\frac{3}{2} \frac{h}{D} \arcsin \frac{h}{D}\right. \\
& \left.+\left(1+\frac{1}{2}\left(\frac{h}{D}\right)^{2}\right) \sqrt{1-\left(\frac{h}{D}\right)^{2}}\right\}
\end{aligned}
$$

Upon insertion of Eqs. (18) and (31) into Eq. (10), we straightforwardly retrieve the Derjaguin expression for the depletion potential of spheres due to discs, Eq. (2). 


\subsection{Exact solution}

We recently published the exact solution of the depletion potential of spheres due to discs elsewhere [19]. Here we give a brief summary of those results up to first order in the density.

The gain in the bulk due to the overlap of the depletion zones is again reflected by Eq. (13) using $\ell=D$

$$
N_{I}=\frac{\pi}{6} n_{p} D^{2} \sigma\left(1-\frac{h}{D}\right)^{2}\left(\frac{3}{2}+\frac{D}{\sigma}+\frac{1}{2} \frac{h}{\sigma}\right) .
$$

The number of particles $N_{I I}$ that fits in the gap between the two spheres, is like Eq. (19) given by the volume integral over the number density of particles that fit in the gap

$$
N_{I I}=4 \pi \int_{\max \left(0, h-\frac{1}{2} D\right)}^{D / 2} \int_{\psi_{i}(x)}^{\psi_{f}(x)}\left(\frac{1}{2} \sigma+x\right)^{2} n(x, \psi) \sin \psi \mathrm{d} \psi \mathrm{d} x .
$$

Here, the angles $\psi$ are again given by Eqs. (21) and (22). The actual number of conformations $n(x, \psi) / n_{p}$ a disc can assume at a given position $x, \psi$ follows from the cones the directors can describe in the vicinity of a sphere, analogously as depicted in Fig. 3

$$
\frac{n(x, \psi)}{n_{p}}=\frac{1}{\pi} \int_{\beta-\theta_{A}}^{\theta_{B}} \sin \theta \arccos \left(\frac{\cos \theta_{A}-\cos \theta \cos \beta}{\sin \theta \sin \beta}\right) \mathrm{d} \theta .
$$

Here $\theta_{A}$ and $\theta_{B}$ give the width of the cones around the normals $\vec{n}$ on both spheres. Moreover, $\beta$ is the angle between these normals. If $\beta+\theta_{A}>\pi-\theta_{B}$ complementary conformations can be assumed, which adds an extra term similar to Eq. (34) with the lower limit set to $\pi-\beta-\theta_{A}$. The angles $\theta$ can be found from

$$
\cos \theta(x)=\left\{\begin{array}{l}
\frac{\sigma}{\sigma+2 x}, \quad x \leqslant x_{0} \\
\sqrt{1-\left\{\frac{\left(\frac{D}{2}\right)^{2}+x(\sigma+x)}{(\sigma+2 x)\left(\frac{D}{2}\right)}\right\}^{2}}, \quad x>x_{0} .
\end{array}\right.
$$

Here we distinguished between the case for $x<x_{0}$, when the face of the disc touches the sphere first, from $x>x_{0}$ when the edge of the disc touches the spheres, where $x_{0}=\frac{1}{2}\left(\sqrt{D^{2}+\sigma^{2}}-\sigma\right)$.

The actual number of discs in the vicinity of the surfaces of two single spheres is like Eq. (29) given by

$$
N_{I I I}=2 n_{p} \int_{\max \left(0, h-\frac{D}{2}\right)}^{D / 2} A(x)\{1-\cos \theta(x)\} \mathrm{d} x .
$$

Here, the area $A(x)$ is given by Eq. (23), whereas the cosine is given by Eq. (35).

The full potential up to first order in density can now be calculated from Eq. (10) using Eqs. (32), (33) and (36). 


\section{Depletion potential for mixtures of spheres and rods}

Finally we consider the depletion potential of two hard spheres of diameter $\sigma$ surrounded by infinitely thin hard rods of length $\ell=L$.

\subsection{Derjaguin approximation}

Like before, the number of rods attained in the bulk by the overlap of depletion zones is given by Eq. (14) using $\ell=L$

$$
N_{I}=n_{r} \frac{\pi}{12} \sigma L^{2} \times 3\left(1-\frac{h}{L}\right)^{2} .
$$

The amount of rods that are still able to access the gap between two large spheres, is formally given by Eq. (19). The areas for the two respective overlap situations are given by Eqs. (25) and (26), where the characteristic size $D$ is replaced by the length $L$. However, the director of the rod points along the rod, whereas it is perpendicular to discs. This affects the expression for the number density, since now the azimuth is given by $\cos \theta_{0}=\frac{x}{L / 2}$ and hence

$$
n(x)=n_{r} \int_{\frac{1}{2} \pi}^{\theta_{0}} \sin \theta \mathrm{d} \theta=n_{r} \frac{x}{L / 2} .
$$

From the aforementioned equations we now find after integration

$$
N_{I I}=\left\{\begin{array}{l}
n_{r} \frac{\pi}{12} \sigma L^{2}\left\{\frac{1}{2}-\left(\frac{h}{L}\right)^{3}\right\}, \quad 0 \leqslant h \leqslant \frac{1}{2} L \\
n_{r} \frac{\pi}{12} \sigma L^{2}\left\{\frac{1}{2}+\frac{1}{2}\left(2 \frac{h}{L}-1\right)^{3}-\left(\frac{h}{L}\right)^{3}\right\}, \quad \frac{1}{2} L<h \leqslant L .
\end{array}\right.
$$

The number of rods near two individual spheres is formally offered by Eq. (29). As before, the area $A_{1}(x)$ follows from Eq. (25) with $D$ replaced by $L$. The number density $n(x)$ is now given by Eq. (38). Integrating provides us with

$$
N_{I I I}=\left\{\begin{array}{l}
n_{r} \frac{\pi}{12} \sigma L^{2}\left\{\frac{5}{2}-3 \frac{h}{L}\right\}, \quad 0 \leqslant h \leqslant \frac{1}{2} L \\
n_{r} \frac{\pi}{12} \sigma L^{2}\left\{\frac{5}{2}-3 \frac{h}{L}+\frac{1}{2}\left(2 \frac{h}{L}-1\right)^{3}\right\}, \quad \frac{1}{2} L<h \leqslant L .
\end{array}\right.
$$

As with the discs, the difference between $N_{I I}$ and $N_{I I I}$ is independent on the two overlap situations

$$
N_{I I}-N_{I I I}=n_{r} \frac{\pi}{12} \sigma L^{2}\left\{-2-\left(\frac{h}{L}\right)^{3}+3 \frac{h}{L}\right\} .
$$

Inserting Eqs. (37) and (41) into Eq. (10) straightforwardly yields the potential of mean force of two spheres in the presence of rods according to the Derjaguin approximation, Eq. (3). 


\subsection{Exact solution}

The exact solution for the depletion potential of spheres in the presence of rods has been scrutinized by Yaman et al. [29]. We benefit from the numerical results of their method $[44,45]$.

The real number of rods that profit in bulk from the overlap of the depletion zones once more follows from Eq. (13) with $\ell=L$

$$
N_{I}=n_{r} \frac{\pi}{12} \sigma L^{2}\left(1-\frac{h}{L}\right)^{2}\left(3+2 \frac{L}{\sigma}+\frac{h}{\sigma}\right) .
$$

The actual amount of rods that approach the two separated large spheres is now formally given by

$$
N_{I I I}=2 n_{r} \int_{\max \left(0, h-\frac{1}{2} L\right)}^{L / 2} A_{1}(x) \cos \theta \mathrm{d} x .
$$

The different cosine term as compared to Eq. (36) is due to the fact that the director of a rod is along the rod instead of perpendicular to it as with discs. Its trigonometric expression should change accordingly. Again we distinguish the case that the face of the rod touches the sphere first $\left(x<x_{0}\right)$ from the case that the edge of the rod touches the spheres $\left(x>x_{0}\right)$

$$
\cos \theta(x)=\left\{\begin{array}{l}
\sqrt{1-\left(\frac{\sigma}{\sigma+2 x}\right)^{2}} \text { if } x \leqslant x_{0} \\
\frac{\left(\frac{1}{2} \sigma+x\right)^{2}+\frac{1}{4}\left(L^{2}-\sigma^{2}\right)}{\left(\frac{1}{2} \sigma+x\right) L}, \quad \text { if } x>x_{0} .
\end{array}\right.
$$

Here $x_{0}=\frac{1}{2}\left(\sqrt{L^{2}+\sigma^{2}}-\sigma\right)$.

The number of rods in the gap between two large spheres, $N_{I I}$, can in principle be given by the same procedure as with discs, Eq. (33). In order to avoid a lengthy calculation and accompanying numerical error, we use the results for the depletion potential of Yaman et al. [29] together with Eqs. (42) and (43) to extract $N_{I I}$ from Eq. (10).

\section{Results and discussion}

The first contribution to the depletion potential, $N_{I}$, stems from the gain of volume in the bulk when the two depletion zones large spheres overlap each other. Since this only depends on the typical size $\ell$ of the depletion agent, these are the same for the small spheres $(\ell=a)$, discs $(\ell=D)$, and rods $(\ell=L)$. The Derjaguin approximation of this contribution is given by Eq. (14). It is drawn by the line in Fig. 4 for small spheres and in Fig. 5(a) and (b) for discs and rods as depletion agents, respectively. We scaled the vertical axis in such a way that the potential equals unity at contact. Moreover, on the horizontal axis the face-to-face separation $h$ is weighted by the characteristic size $\ell$ of the depletion agent such that the reduced distance runs from 0 to 1 . Here we have 


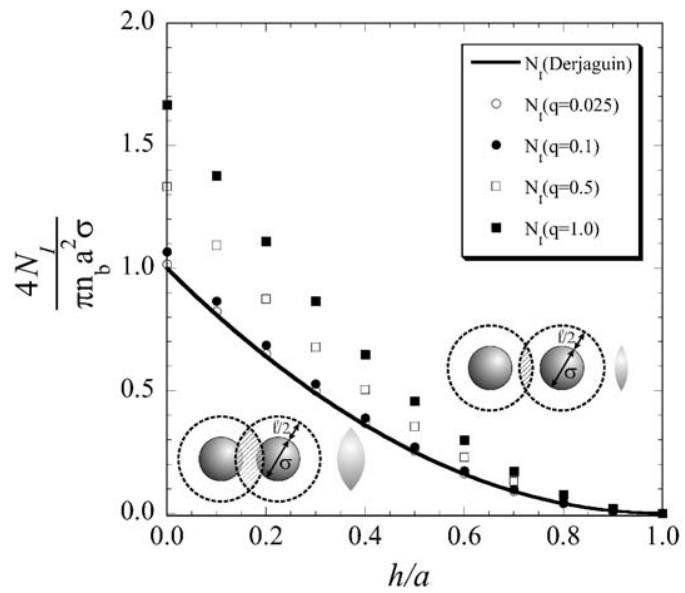

Fig. 4. The reduced number of small spheres of diameter $a$ that gains accessible volume when two depletion zones of the big spheres of diameter $\sigma$ overlap. The line gives the Derjaguin prediction, Eq. (14), the symbols the actual number from Eq. (13) as a function of the size ratio $q \equiv a / \sigma$.

derived the Derjaguin approximation by neglecting higher order terms in the curvature of the exact overlap volume. As a consequence, the size of the overlap volume is underestimated which gives rise to a lower estimate of $N_{I}$ than in the exact solution, Eq. (13). The exact solutions are given by the symbols in Fig. 4 and Fig. 5(a) and (b) as a function of the size ratio $q \equiv \ell / \sigma$.

As expected, the deviation of the Derjaguin approximation from the exact solution increases with increasing size ratio. The error introduced by the Derjaguin approximation is straightforwardly found from Eqs. (13) and (14). At contact $(h=0)$, where the deviation from the exact solution is the largest, the error is given by

$$
1-\frac{N_{I}^{\text {Derjaguin }}}{N_{I}^{\text {exact }}}=\frac{1}{\frac{3}{2} \frac{\sigma}{\ell}+1} .
$$

For $\sigma \gg \ell$ we indeed find that the Derjaguin approximation goes to the exact solution.

The number of discs or rods that still fit in the gap between two spheres, $N_{I I}$, is given in Fig. 5(c) and (d), respectively. Like $N_{I}$, the curvature of the actual volume provides more particles to fit in the edges than accounted for in the Derjaguin approximation. Hence, it again underestimates the real number of particles that still fit in the gap. Since the excluded volume of a disc is larger than that of a rod at the same characteristic size $\ell$, the number of conformations a disc can assume when confined between two spheres is much lower than that of rods. This is basically why the $N_{I I}$ 's of either shapes differ with one order of magnitude.

Upon larger separation the number of conformations initially increases apparently faster than the decrease of the overlap volume, since the exact $N_{I I}$ 's go through a maximum. This feature is missed in the Derjaguin approximation since there the initial increase of conformations goes almost linearly with the decrease of volume (cf. Eq. (27) and Eqs. (25) and (26)). We established that a secondary minimum (larger 

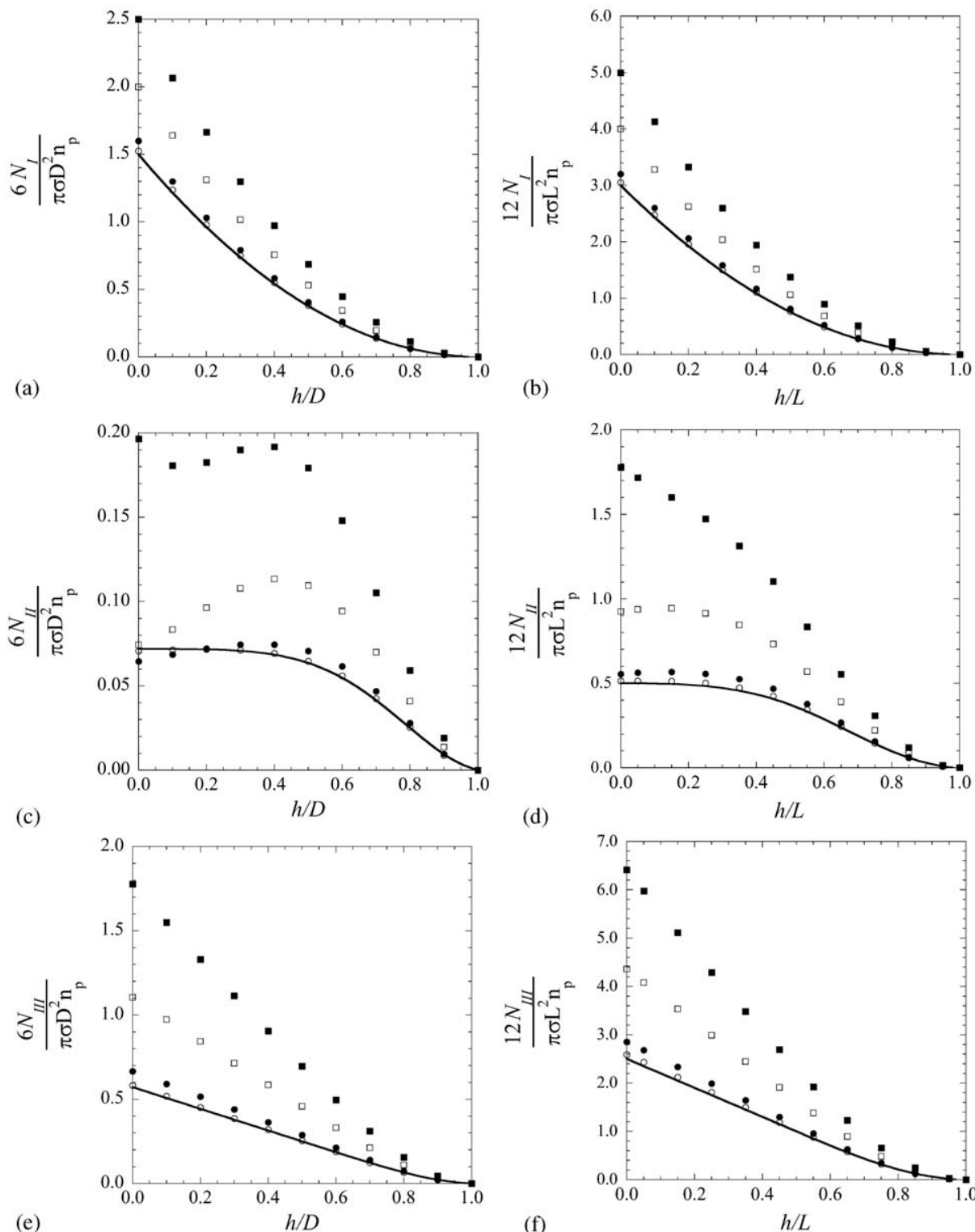

Fig. 5. The three contributions to the depletion potential for discs (left column) and rods (right). The first row shows the reduced number of (a) discs and (b) rods that gain accessible volume in the bulk due to the overlap of depletion zones. The row in the middle gives the reduced number of particles that are between the two large spheres for (c) discs and (d) rods. The last row presents the reduced number of particles in the vicinity of two separated spheres for (e) discs and (f) rods. The drawn lines give the Derjaguin approximation, Eq. (14), Eqs. (28) and (39), and Eqs. (30) and (40), respectively, whereas the symbols give the exact solution for several size ratios $q \equiv \ell / \sigma(\bigcirc: q=0.025, \boldsymbol{O}: q=0.1, \square: q=0.5, \mathbf{\square}: q=1.0)$. 

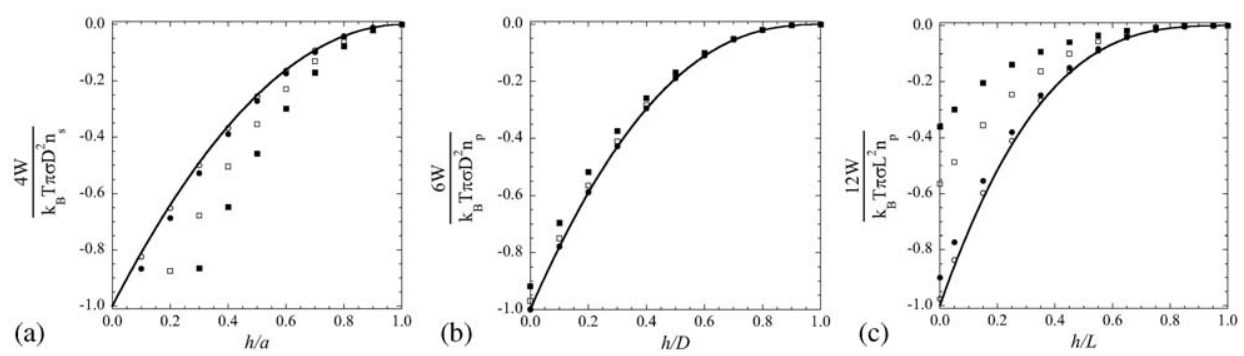

Fig. 6. The reduced depletion potentials of spheres due to (a) small spheres $(\ell=a)$, (b) discs $(\ell=D)$, and (c) rods $(\ell=L)$. The drawn lines give the Derjaguin approximation, as they follow from (a) Eq. (1), (b) Eq. (2), and (c) Eq. (3), respectively. The symbols denote the exact solutions as they follow from (a) Vrij [17], (b) our previous work [19], and (c) Yaman et al. [29] for several size ratios $q \equiv \ell / \sigma$ (○: $q=0.025, \bigcirc: q=0.1, \square: q=0.5, \mathbf{\square}: q=1.0$ ).

than the numerical error) in $N_{I I}$ of discs slowly enters for $q \gtrsim 0.75$. This pronounced secondary minimum is observed in Fig. 5(c) for $q=1.0$.

A similar reasoning can be held for $N_{I I I}$, the number of particles that access the overlap volume when the two large spheres are 'inifinitely' apart, i.e., $h>\ell . N_{I I I}$ for discs and rods are shown in Fig. 5(e) and (f), respectively. The number of particles in the real overlap volume is once more higher than in the truncated volume of the Derjaguin approximation. Because of the larger excluded volume of the discs, their $N_{I I I}$ is again lower than that of the rods. In the vicinity of a single sphere there are more possible conformations then confined between two spheres, so $N_{I I I}$ is larger than $N_{I I}$ and of the same order magnitude as $N_{I}$.

Having all three contributions, we obtain from Eq. (10) the depletion potential of large spheres, as depicted in Fig. 6. As previously has been found, the Derjaguin approximation for small spheres underestimates the true potential, whereas it overestimates the interaction due to rods. For discs the Derjaguin approach turns out to be surprisingly accurate. With the three contributions at hand we now can argue where the precision stems from.

For small hard spheres the Derjaguin approximation for $N_{I I}$ and $N_{I I I}$ are exact, i.e., no small sphere can enter the depletion zones of the large spheres, irrespective of their mutual distance. Consequently, the error in the Derjaguin approach of the depletion potential of large spheres due to small spheres follows from Eq. (45) and is always underestimated by the Derjaguin approximation, as depicted in Fig. 6(a). Up to first order in the density this is also valid for polymer coils.

For discs and rods $N_{I I}$ and $N_{I I I}$ look qualitatively the same. The relative error of the Derjaguin approximation in $N_{I I}$ and $N_{I I I}$ for discs and rods are alike for the same characteristic sizes. For either shapes this error increases up to about $66 \%$ of the exact values for a unit size ratio. From this point of view it is far from obvious why the Derjaguin approximation works out that well for discs. A disc looks from its topview identical to a sphere of the same dimension, whereas from its sideview it is indistinguishable from a like-sized infinitely thin rod. The intermediate excluded volume 
of a disc close to a sphere counterbalances the underestimation of the freely rotating one in the bulk. Due to the relatively large excluded volume of rods near a sphere, the number of rods in the edges of the overlap volume is severely underestimated by the Derjaguin approximation which subsequently lead to an overestimation of the true potential. Hence, the 'sphere-like' behaviour of a freely rotating disc is just compensated by the 'rod-like' manner it conducts near a wall. Although from this point of view it is not surprising the Derjaguin approximation for discs is most accurate, we are astonished that it is that precise.

In this paper we have shown that the Derjaguin expressions for the depletion potential can be recovered by considering three contributions to the depletion effect in the limit of small depletion agents. Moreover, we also obtained three exact formulations up to first order in the number density. By comparison of the contributions we grasp that the excluded volume of the depletion agents determines the accuracy of the Derjaguin approximation for the total potential. The separate contributions nevertheless show the shortcomings of the Derjaguin approximation for increasing aspect ratios. From the approach presented by Henderson [46] as well as ours, we conclude that one may rely on the Derjaguin approach for depletion forces within the appropriate limits, i.e., dilute suspensions of relatively small depletion agents.

\section{Acknowledgements}

We are grateful to Dr. C.M. Marques of Université Louis Pasteur, Strasbourg, for providing us with the data of the sphere/rod potential. This work is part of the SoftLink research programme of the 'Stichting voor Fundamenteel Onderzoek der Materie (FOM)', which is financially supported by the 'Nederlandse Organisatie voor Wetenschappelijk Onderzoek (NWO)'.

\section{References}

[1] S. Sanyal, N. Easwar, S. Ramaswamy, A.K. Sood, Europhys. Lett. 18 (1992) 107.

[2] J.S. van Duijneveldt, A.W. Heinen, H.N.W. Lekkerkerker, Europhys. Lett. 21 (1993) 369.

[3] P.D. Kaplan, J.L. Rouke, A.G. Yodh, D.J. Pine, Phys. Rev. Lett. 72 (1994) 582.

[4] A.D. Dinsmore, A.G. Yodh, D.J. Pine, Phys. Rev. E 52 (1995) 4045.

[5] U. Steiner, A. Meller, J. Stavans, Phys. Rev. Lett. 74 (1995) 4750.

[6] A. Imhof, J.K.G. Dhont, Phys. Rev. Lett. 75 (1995) 1662.

[7] T. Biben, J.P. Hansen, Phys. Rev. Lett. 66 (1991) 2215.

[8] T. Biben, J.P. Hansen, J. Phys.: Condens. Matter 3 (1991) 65.

[9] H.N.W. Lekkerkerker, A. Stroobants, Physica A 195 (1993) 387.

[10] W.C.K. Poon, P.B. Warren, Europhys. Lett. 28 (1994) 513.

[11] Y. Rosenfeld, Phys. Rev. Lett. 72 (1994) 3831.

[12] M. Dijkstra, R. van Roij, R. Evans, Phys. Rev. Lett. 81 (1998) 2268.

[13] M. Dijkstra, R. van Roij, R. Evans, Phys. Rev. Lett. 82 (1999) 117.

[14] M. Dijkstra, R. van Roij, R. Evans, Phys. Rev. E 59 (1999) 5744.

[15] S. Asakura, F. Oosawa, J. Chem. Phys. 22 (1954) 1255.

[16] Y. Mao, M.E. Cates, H.N.W. Lekkerkerker, Physica A 222 (1995) 10.

[17] A. Vrij, Pure Appl. Chem. 48 (1976) 471. 
[18] M. Piech, J.Y. Walz, J. Colloid Interface Sci. 232 (2000) 86.

[19] S.M. Oversteegen, H.N.W. Lekkerkerker, Phys. Rev. E 68 (2003) 021404.

[20] M. Adams, Z. Dogic, S.L. Keller, S. Fraden, Nature 393 (1998) 349.

[21] G.H. Koenderink, G.A. Vliegenthart, S.G.J.M. Kluijtmans, A. van Blaaderen, A.P. Philipse, H.N.W. Lekkerkerker, Langmuir 15 (1999) 4683.

[22] G.A. Vliegenthart, A. van Blaaderen, H.N.W. Lekkerkerker, Faraday Discuss. 112 (1999) 173.

[23] P.G. Bolhuis, D. Frenkel, J. Chem. Phys. 101 (1994) 9869.

[24] G.A. Vliegenthart, H.N.W. Lekkerkerker, J. Chem. Phys. 111 (1999) 4153.

[25] Z. Dogic, D. Frenkel, S. Fraden, Phys. Rev. E 62 (2000) 3925.

[26] L. Auvray, J. Phys. (Paris) 42 (1981) 79.

[27] Y. Mao, M.E. Cates, H.N.W. Lekkerkerker, Phys. Rev. Lett. 75 (1995) 4548.

[28] Y. Mao, M.E. Cates, H.N.W. Lekkerkerker, J. Chem. Phys. 106 (1997) 3721.

[29] K. Yaman, C. Jeppesen, C.M. Marques, Europhys. Lett. 42 (1998) 221.

[30] K. Lin, J.C. Crocker, A.C. Zeri, A.G. Yodh, Phys. Rev. Lett. 87 (2001) 088301.

[31] K. Lin, J.C. Crocker, A.C. Zeri, A.G. Yodh, Phys. Rev. Lett. 87 (2001) 269902.

[32] L. Helden, R. Roth, G.H. Koenderink, P. Leiderer, C. Bechinger, Phys. Rev. Lett. 90 (2003) 048301.

[33] B.V. Derjaguin, Kolloid-Z. 69 (1934) 155.

[34] D.G. Hall, J. Chem. Soc. Faraday Trans. 168 (1972) 2169.

[35] S.G. Ash, D.H. Everett, C. Radke, J. Chem. Soc. Faraday Trans. 169 (1973) 1256.

[36] R. Evans, B. Marini, U. Marconi, J. Chem. Phys. 86 (1987) 7138.

[37] Y. Mao, P. Bladon, H.N.W. Lekkerkerker, M.E. Cates, Mol. Phys. 92 (1997) 151.

[38] W.B. Russel, D.A. Saville, W.R. Schowalter, Colloidal Dispersions, Cambridge University Press, New York, 1989, pp. 149-150.

[39] J. Lyklema, Fundamentals of Interface and Colloid Science, Vol. I, Academic Press, London, 1991, pp. 4.61-4.64.

[40] R.J. Hunter, Foundation of Colloid Science, 2nd Edition, Oxford University Press, Oxford, 2001, pp. 549-552.

[41] J.M. Brader, R. Evans, Physica A 306 (2002) 287.

[42] S.M. Oversteegen, H.N.W. Lekkerkerker, Physica A 310 (2002) 181.

[43] H.N.W. Lekkerkerker, S.M. Oversteegen, J. Phys.: Condens. Matter 14 (2002) 9317.

[44] K. Yaman, P. Pincus, C.M. Marques, Phys. Rev. Lett. 78 (1997) 4514.

[45] K. Yaman, M. Jeng, C. Jeppesen, P. Pincus, C.M. Marques, Physica A 247 (1997) 159.

[46] J.R. Henderson, Physica A 313 (2002) 321. 